\title{
Nickel-reduced Graphene Oxide-Cellulose Nanofiber Composite Papers for Electromagnetic Interference Shielding
}

\author{
Zhibing Chen, Qinqin Zhang, Wanyao Meng, Zhenxing Wang, Xuewen Han, and \\ Junwen $\mathrm{Pu}$ *
}

\begin{abstract}
With the widespread use of electronic communication devices, ultra-thin, flexible, and high-performance electromagnetic interference (EMI) shielding materials are widely used to prevent radiation pollution. Moreover, graphene-reinforced polymer-based nanocomposites have received increasing attention in the field of EMI shielding because of their excellent electrical properties. In this work, ultrathin and flexible nickel/reduced graphene oxide/cellulose nanofiber (Ni/RGO/CNFs) composite paper was fabricated using a step-by-step vacuum-assisted filtration method. The prepared composite paper with Ni/RGO to CNF ratio of 3:1 and a thickness of $105 \mu \mathrm{m}$ attained the electrical conductivity of $1664 \mathrm{~S} / \mathrm{m}$ and $22.6 \mathrm{~dB}$ of excellent electromagnetic shielding effectiveness (SE). In addition, the composite paper (2:1) exhibited great mechanical properties, leading to a high tensile strength of $78.4 \mathrm{MPa}, 10.3 \%$ fracture strain, and no obvious fractures or cracks when the composite paper was folded. Therefore, the Ni/RGO/CNFs composite paper prepared in this study is an effective lightweight shielding material, especially in flexible electronics and wearable devices.
\end{abstract}

Keywords: Cellulose nanofiber; Reduced graphene oxide; Nickel; Electromagnetic interference shielding

Contact information: MOE Engineering Research Center of Forestry Biomass Materials and Bioenergy, Beijing Forestry University, Beijing, 100083, PR China; *Corresponding author: jwpu@bjfu.edu.cn

\section{INTRODUCTION}

With the widespread application of wireless communication technology and use of electronic devices, electromagnetic (EM) radiation pollution is aggravated, which not only affects the normal operations of electronic devices, but is also seriously harmful to human health. Therefore, the development of lightweight and efficient electromagnetic interference (EMI) shielding materials is essential to solve electromagnetic radiation pollution (Chen et al. 2016; Cao et al. 2018). In recent years, conductive polymer composites (CPCs) have attracted extensive consideration in electromagnetic shielding applications. In particular, carbon-based (e.g., graphene, reduced graphene oxide, carbon nanotubes, etc.) conductive polymer composites have received considerable attention, which is different from traditional metal-based materials. These materials have the advantages of flexible designing, easy processing, corrosion resistance, low density, etc. Carbon-based materials are expected to be widely used as candidates for ultrathin electromagnetic shielding materials with excellent electromagnetic shielding properties (Remyamol et al. 2013; Prasad et al. 2018).

Graphene, two-dimensional (2D) carbon nanofillers, have an extremely high conductivity and excellent EMI shielding performance (Geim and Novodelov 2007; 
Bansala et al. 2019). Polymer composites with simple graphene are difficult to achieve EMI shielding efficiency (SE) values over a wide frequency band. Combining graphene with magnetic materials (e.g., iron, cobalt, nickel and its oxides, etc.) not only enhances shielding effectiveness, but also broadens the shielding band (Tian et al. 2017; Huangfu et al. 2019; Jagdees et al. 2019). However, the strong Van der Waals interactions between graphene makes it difficult to uniformly disperse into the matrix, especially at high loads, which limits its practical application. To restore the $\mathrm{sp}^{2}$ hybridized structure of graphene and remove defects, the graphene oxide (GO) is usually used after reduction. Xu et al. (2018) prepared a super-flexible graphene/polydimethylsiloxane (PDMS) nanocomposite for high-performance EMI shielding. When the graphene loading is at $3.07 \mathrm{wt} \%$ level, the conductivity of the nanocomposite reaches up to $103 \mathrm{~S} / \mathrm{m}$, the EMI shielding performance in the X-band reached approximately $54 \mathrm{~dB}$, and approximately $99.999 \%$ shielding efficiency was achieved. As the most abundant and renewable natural polymer resource, cellulose and its derivatives have attracted widespread attention. Cellulose nanofiber (CNF), as a cellulose derivative, is often used as a polymer matrix because of its good mechanical properties, and it can be uniformly dispersed in aqueous solution (Isogai et al. 2011; Klemm et al. 2011). More importantly, compared with other polymers, the onedimensional (1D) nano-fibrous structure of CNF would result in less insulating contact between the 2D graphene nanosheets. Typically, conductive polymer composites (CPCs) are fabricated by introducing graphene into the polymer matrix via several processing strategies, including melt blending, solution mixing, in situ polymerization, etc. Yang et al. (2017) prepared reduced graphene oxide (RGO) - cellulose nanofiber (CNF) composite films via a standard vacuum filtration process followed by a post-reduction step. The asprepared RGO/CNF foam exhibited excellent EMI SE of $26.2 \mathrm{~dB}$ at a lower thickness of $23 \mu \mathrm{m}$ and an RGO loading of $50 \mathrm{wt} \%$. There are only few studies available in the literature on the adhesion of metals on graphene and the formation of shielding materials by a composite with CNF (Xu et al. 2008; Prasad et al. 2019).

In this paper, the authors prepared an ultrathin and flexible $\mathrm{Ni} / \mathrm{RGO} / \mathrm{CNF}$ composite paper possessing a sandwich structure by a simple step-by-step vacuum filtration. The prepared composite paper exhibited excellent electrical conductivity and EMI shielding efficiency, good mechanical and thermodynamic properties, and can be applied to wearable, portable equipment as well as conductive materials.

\section{EXPERIMENTAL}

\section{Materials}

The GO was supplied by Beijing Carbon Century Co., Ltd. (Beijing, China). Ethylene glycol and sodium hydroxide were purchased from Beijing Chemical Works (Beijing, China). Nickel chloride hexahydrate was purchased from Tianjin Yongda Chemical Regent Co., Ltd. (Tianjin, China). The cellulose nanofibers were provided by Tianjin Woodelfbio Cellulose Co., Ltd. (Tianjin, China). Hydrazine hydrate was obtained from Shanghai Aladdin Bio-Chem Technology Co., Ltd. (Shanghai, China). All other materials were used as received without further purification.

\section{Preparation of $\mathrm{Ni} / \mathrm{RGO}$}

First, $50 \mathrm{mg}$ GO and $400 \mathrm{mg}$ nickel chloride hexahydrate were dissolved in a waterethylene glycol $(60 \mathrm{~mL})$ mixture to form a homogeneous solution by mechanical stirring 
at a speed of $900 \mathrm{rpm}$ for $30 \mathrm{~min}$. Then, $17 \mathrm{mg}$ D-glucose was added to the dispersion and ultra-sonication was continued for $1 \mathrm{~h}$. Finally, hydrazine hydrate solution containing 0.5 $\mathrm{g}$ of sodium hydroxide was added and the dispersion was transferred to a Teflon-lined autoclave after stirring for $10 \mathrm{~min}$. The contents were autoclaved at $200{ }^{\circ} \mathrm{C}$ for $80 \mathrm{~min}$. Next, it was removed and allowed to naturally cool to room temperature. The dark precipitate obtained was washed several times with ethanol and deionized water, then filtered, and the end product was dried at a $60{ }^{\circ} \mathrm{C}$ for at least $12 \mathrm{~h}$.

\section{Preparation of $\mathrm{Ni} / \mathrm{RGO} / \mathrm{CNF}$ composite papers}

The Ni/RGO/CNF composite papers with a sandwich structure were fabricated by a step-by-step vacuum filtration process, which is assembled from a sand core funnel, filter bottle, and holder. The filtration process is carried out at room temperature and the dispersion is alkaline. Two equal masses of dried $\mathrm{Ni} / \mathrm{RGO}$ nanoparticles were weighed and dispersed in deionized water separately. A $0.1 \mathrm{wt} \% \mathrm{Ni} / \mathrm{RGO}$ dispersion was filtered through a polyvinylidene fluoride microporous membrane ( $0.45 \mathrm{um}$ pore size, $50 \mathrm{~mm}$ in diameter) to form a first layer. Then, a $0.1 \mathrm{wt} \% \mathrm{CNF}$ suspension was slowly added and ensured that the CNF was uniformly covered on the Ni/RGO surface. Finally, an equal amount and concentration of $\mathrm{Ni} / \mathrm{RGO}$ dispersion was added to form the last layer. After the water was filtering out, the composite paper with three layers was dried at room temperature for at least $12 \mathrm{~h}$. The $\mathrm{Ni} / \mathrm{RGO} / \mathrm{CNF}$ composite papers with different weight ratio of $\mathrm{Ni} / \mathrm{RGO}$ to CNF $(1: 1,2: 1,3: 1$, and 4:1) were successfully prepared as described above. In addition, pure CNF paper was also prepared under the same conditions and used for comparative studies.

\section{Methods}

\section{Characterization}

Microscopic morphologies of samples were investigated using field-emission scanning electron microscopy and energy dispersive spectroscopy (FE-SEM and EDS; S4800; Hitachi, Tokyo, Japan). Thermogravimetric analysis (TGA) was performed on a simultaneous thermal analyzer (TGA/DSC 1/1600; Mettler-Toledo, Zurich, Switzerland). Raman spectra were recorded via a reflective Raman system with an excitation laser of 532 nm (LabRAM ARAMIS; HORIBA Jobin Yvon, Paris, France). The X-ray diffraction (XRD) data were recorded on an X-ray diffractometer (D8 Advance; Bruker, Karlsruhe, Germany). The Fourier transform infrared (FTIR) spectra were obtained using a Nicolet iS10 (Thermo Fisher, Waltham, MA, USA) spectrometer. The mechanical properties of the $\mathrm{Ni} / \mathrm{RGO} / \mathrm{CNF}$ composite paper were measured using a universal testing machine (CMT6103; MTS, Minneapolis, MN, USA). Each sample was cut into strips of $10 \mathrm{~mm} \times$ $30 \mathrm{~mm}$ dimensions, and the loading rate used was $0.2 \mathrm{~mm} / \mathrm{min}$. The thickness of the composite paper was measured by a Vernier caliper. The electrical conductivity measurements were performed at room temperature using the standard four-probe method on a physical property measurement system (ST2258C; JG, Suzhou, China). At least three samples were tested for each measurement to guarantee the accuracy of the data.

To obtain the EMI SE values of composite papers, the scattering parameters $\left(S_{11}\right.$ and $S_{21}$ ) were measured by a vector network analyzer (PNA-N5244A; Agilent Technologies, Palo Alto, CA, USA) using the waveguide method within $8.2 \mathrm{GHz}$ to 12.4 GHz. All the samples were cut into rectangle-shaped specimens of $22.9 \mathrm{~mm} \times 10.2 \mathrm{~mm}$ for measurements. The vector network analyzer was calibrated before measurement. When the incident EM wave arrives on the surface of materials, the incident power $\left(P_{\mathrm{i}}\right)$ is divided 
into the reflected power $\left(P_{\mathrm{r}}\right)$, the absorbed power $\left(P_{\mathrm{a}}\right)$, and the transmitted power $\left(P_{\mathrm{t}}\right)$, corresponding to the reflectivity coefficients $(R)$, transmissivity coefficients $(T)$, and absorption coefficients $(A)$. These coefficients can be calculated from the scattering parameters $\left(S_{11}, S_{22}, S_{21}\right.$, and $\left.S_{12}\right)$ and their relationship can be expressed as follows (Panigrahi and Srivastava 2015):

$$
\begin{aligned}
& R+A+T=1 \\
& R=\left|S_{11}\right|^{2}=\left|S_{22}\right|^{2} \\
& T=\left|S_{21}\right|^{2}=\left|S_{12}\right|^{2}
\end{aligned}
$$

The total EMI shielding effectiveness $\left(S E_{\mathrm{T}}\right)$ of the composite paper is the sum of microwave absorption $\left(S E_{\mathrm{A}}\right)$, microwave reflection $\left(S E_{\mathrm{R}}\right)$, and microwave multiple internal reflections $\left(S E_{\mathrm{M}}\right)$, and the equations are as follows (Bian et al. 2017):

$$
\begin{aligned}
& S E_{\mathrm{T}}=S E_{\mathrm{A}}+S E_{\mathrm{R}}+S E_{\mathrm{M}} \\
& S E_{\mathrm{R}}=-10 \log (1-R) \\
& S E_{\mathrm{A}}=-10 \log \left(\frac{T}{1-R}\right)
\end{aligned}
$$

When $S E_{\mathrm{T}} \geq 10 \mathrm{~dB}$, the $S E_{\mathrm{M}}$ can be ignored (Hsiao et al. 2014). The EMI shielding efficiency (\%) is calculated using Eq. 7 (Cao et al. 2018):

$$
\text { EMI shielding efficiency }(\%)=100-\frac{1}{10^{S E / 10}} \times 100(\%)
$$

\section{RESULTS AND DISCUSSION}

Figure 1a shows the overall fabrication process for the preparation of the $\mathrm{Ni} / \mathrm{RGO} / \mathrm{CNFs}$ composite paper. Previous study has indicated that the water-ethylene glycol mixture is beneficial for the synthesis of graphene-metal particle nanocomposites (Xu et al. 2008). Therefore, the Ni/RGO nanoparticles were prepared by hydrothermal method using a mixture of ethylene glycol and water as a solvent medium and hydrazine hydrate as a reducing agent. The next step was the preparation of sandwich structure for composite paper via a step-by-step vacuum filtration (Hou et al. 2018) method. The composite papers with various $\mathrm{Ni} / \mathrm{RGO}$ loading levels were prepared successfully.

In order to detect the degree of reduction of graphene oxide, Raman spectra was used to analyze graphene oxide and Ni/RGO particles. The D-band at about $1345 \mathrm{~cm}^{-1}$ is originated from the disorder and defects of graphene, G band at about $1587 \mathrm{~cm}^{-1}$ is usually attributed to the in-plane stretching vibrations of $\mathrm{sp}^{2}$ hybrid mode carbon atom. The intensity ratio ID/IG can be used to confirm the degree of defect in graphene. As shown in Fig. $1 \mathrm{~b}$, according to calculation, the values of ID/IG ratio of GO and Ni/RGO were 0.89 and 1.09 , which reveals the recovery of the $\mathrm{sp} 2$ carbon domains and an increase in structural defects during the reduction process.

The microstructure of the fracture surface of the $\mathrm{Ni} / \mathrm{RGO} / \mathrm{CNFs}$ composite paper was observed by SEM. Figure 1c shows the microstructure of the cross-section of the composite paper. Through the cohesion and support of CNFs, the Ni/RGO nanosheets formed an arrangement in the plane direction, which ensured good connection of $\mathrm{Ni} / \mathrm{RGO}$ nanosheets and formed a good electrical conductivity network. 
(a)

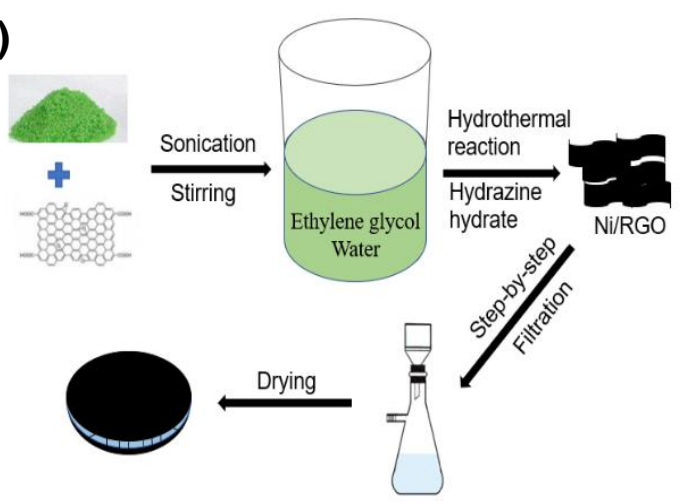

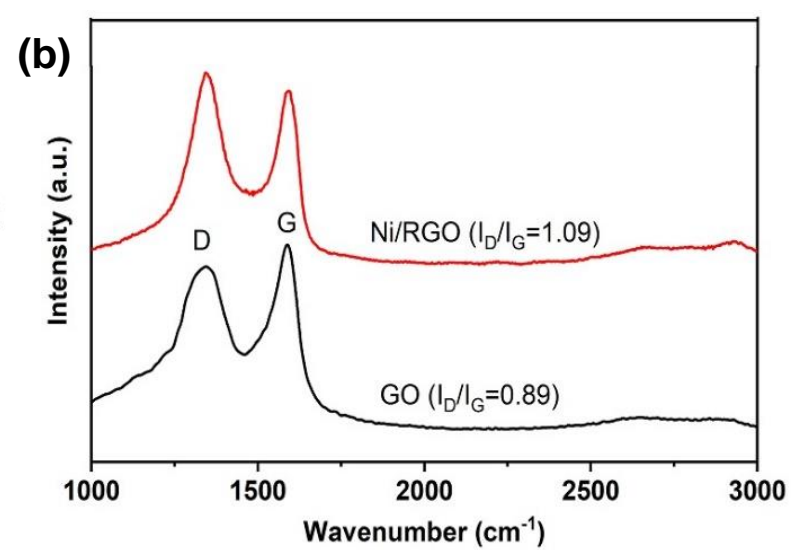

(c)
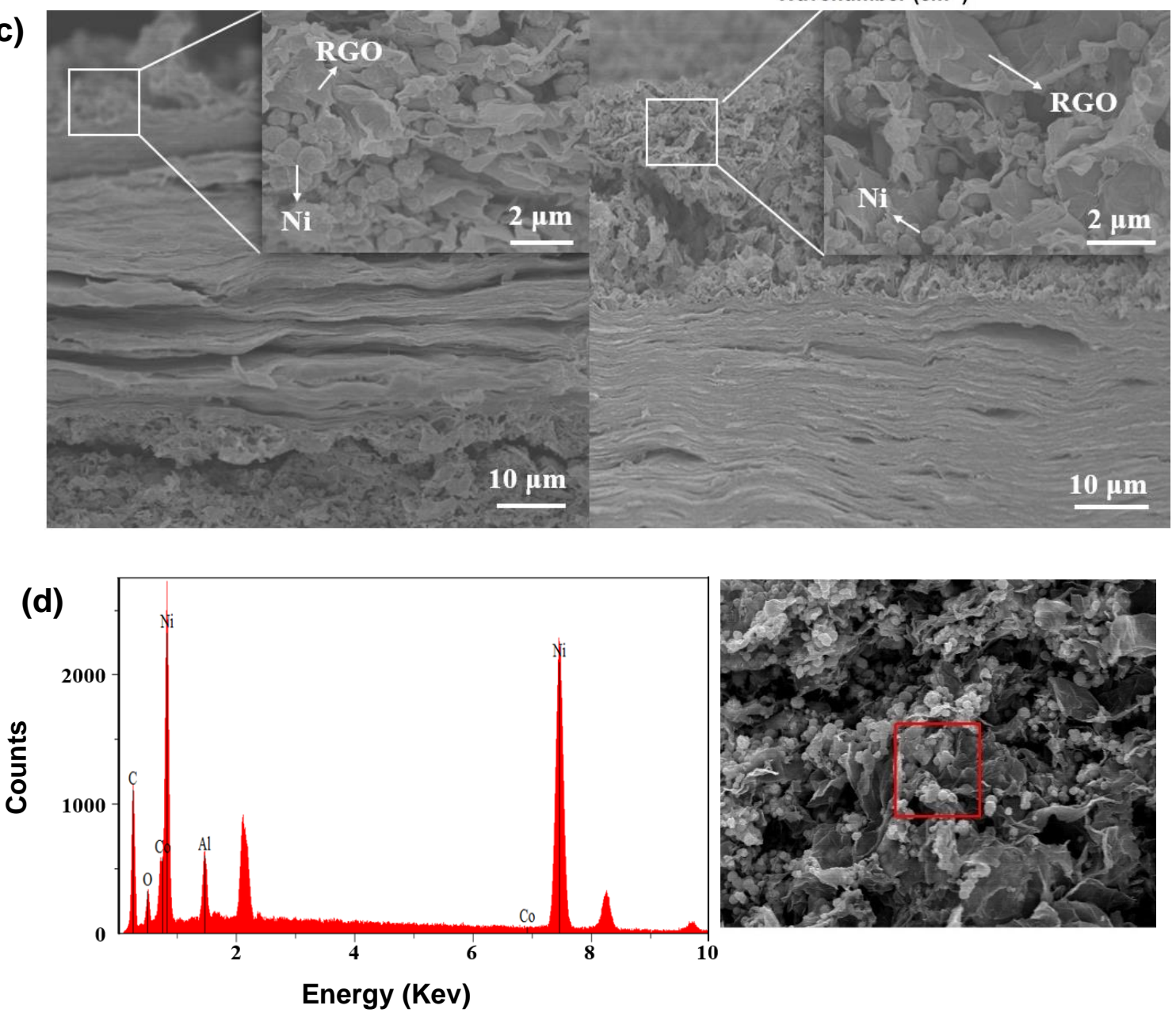

Fig. 1. (a) Schematic of the fabrication process of the Ni/RGO/CNF composite paper;(b) Raman spectra of GO and Ni/RGO; (c) Cross-sectional SEM images of the composite paper with $\mathrm{Ni} / \mathrm{RGO}$ to CNF ratio of 2:1 and 4:1; (d) EDS spectroscopy of a point in the cross section of the composite paper with $\mathrm{Ni} / \mathrm{RGO}$ to CNF ratio of $4: 1$.

In addition, it was clear from Fig. 1c that the composite paper had an ordered lamellar structure without any preferential orientation when the ratio of $\mathrm{Ni} / \mathrm{RGO}$ to $\mathrm{CNF}$ in the composite paper were 2:1 and 4:1. Spherical nickel particles were widely distributed on the graphene surface, and no noticeable agglomeration effects were observed on the 
conductive network. Different from other polymers, 1D CNF can promote the formation of a layered structure of graphene and serve as a matrix for $\mathrm{Ni} / \mathrm{RGO}$ film formation. Figure $1 \mathrm{c}$ also indicates that the $\mathrm{Ni} / \mathrm{RGO}$ nanosheets along the planar direction were supported by CNFs. When the Ni/RGO ratio in the composite paper is increased from $2: 1$ to $4: 1$, the structure of the composite paper becomes denser due to the increased permeability of the $\mathrm{Ni} /$ RGO particles, and the thickness of the upper and lower layers also increased obviously. This type of layered structure and increased thickness of the conductive layer were beneficial to the electromagnetic wave reflection and absorption processes. In addition, EDS was used to test sample composition and content. Figure 1d shows the element and content of a point in the cross section of the composite paper when the ratio of $\mathrm{Ni} / \mathrm{RGO}$ to CNFs was $4: 1$. The content of nickel is about $26.55 \%$, the content of $\mathrm{C}$ element is about $58.61 \%$, the content of $\mathrm{O}$ element is about $8.96 \%$, and a small amount of other elements are negligible. It is also a good illustration of the reduction of graphene oxide and the introduction of nickel.

The FTIR spectroscopy was used to detect the changes in chemical functional groups after compounding reduction of graphene oxide and CNF. The FTIR spectra of the pure Ni/RGO, pure CNFs, and Ni/RGO/CNFs (2:1) composite paper are shown in Fig. 2a. For pure CNFs, absorption peaks were found at $3340 \mathrm{~cm}^{-1}$ and $1617 \mathrm{~cm}^{-1}$ (-OH bending), $2915 \mathrm{~cm}^{-1}$ (C-H stretching), $1060 \mathrm{~cm}^{-1}$ (C-O stretching), $667 \mathrm{~cm}^{-1}$ (-OH out-of-plane bending). The Ni/RGO nanoparticles displayed three inconspicuous peaks at $1700 \mathrm{~cm}^{-1}$, $3667 \mathrm{~cm}^{-1}$, and $1052 \mathrm{~cm}^{-1}$, corresponding to the absorptions of surface terminal groups of $\mathrm{C}=\mathrm{O},-\mathrm{OH}$, and epoxy $\mathrm{C}-\mathrm{O}$, respectively. These stretching vibration peaks were very weak, which indicated that the GO was generally reduced by hydrazine, and the presence of nickel nanoparticles did not hinder the reduction of GO. After compounding Ni/RGO and CNFs, the prepared composite paper exhibited characteristic absorption bands at $2930 \mathrm{~cm}^{-1}(\mathrm{C}-\mathrm{H}$ stretching), $3667 \mathrm{~cm}^{-1}$ (-OH bending), and $1702 \mathrm{~cm}^{-1}$ ( $\mathrm{C}=\mathrm{O}$ stretching). However, when the composite paper was prepared by a step-by-step vacuum filtration method, these characteristic peaks were found to be very weak.
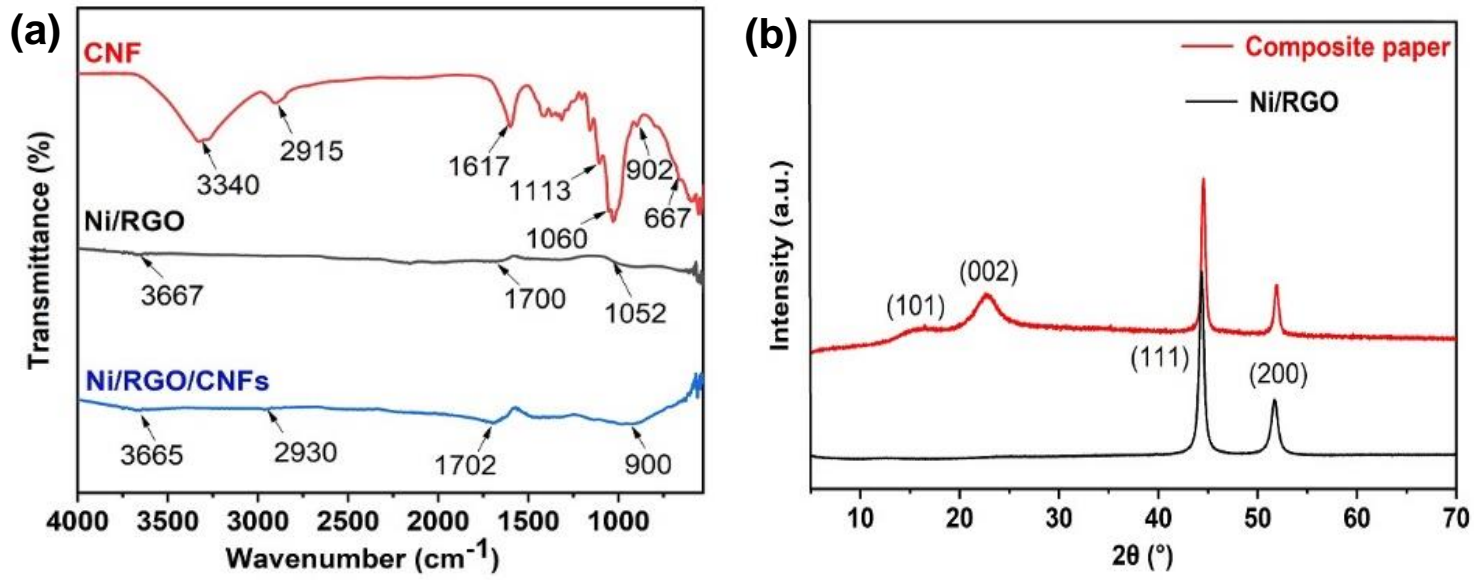

Fig. 2. (a) FTIR spectra of the Ni/RGO, pure CNFs, and Ni/RGO/CNFs (3:1) composite paper; (b)XRD patterns of the Ni/RGO and Ni/RGO/CNFs composite paper (2:1)

To investigate the chemical nature and crystalline structure, spectroscopic analysis was performed using XRD. Figure. $2 \mathrm{~b}$ shows the XRD patterns of the pure CNF papers, pure $\mathrm{Ni} / \mathrm{RGO}$, and $\mathrm{Ni} / \mathrm{RGO} / \mathrm{CNFs}$ composite paper with a ratio of $2: 1$. However, no 
obvious diffraction peaks of graphene oxide were observed, which indicated that most of the oxygen-containing groups of graphene oxide that were reduced by hydrazine hydrate disappeared. In addition, the characteristic peaks at $2 \theta$ values of $44.52^{\circ}$ and $51.74^{\circ}$ corresponding to (111) and (200) crystal planes, respectively, of nickel were seen, implying the successful conversion of $\mathrm{Ni}$ to $\mathrm{Ni} / \mathrm{RGO}$ particles. The peak corresponding to the $2 \theta$ value of $16.5^{\circ}$ in the composite paper was associated to the (101) crystal plane of celluloseI, and the diffraction peak at $2 \theta=22.5^{\circ}$ corresponds to the (002) crystal plane of celluloseI crystalline structure. In addition, with the increasing contents of the CNFs in the composite papers, the two representative peaks of CNFs became increasingly distinct.
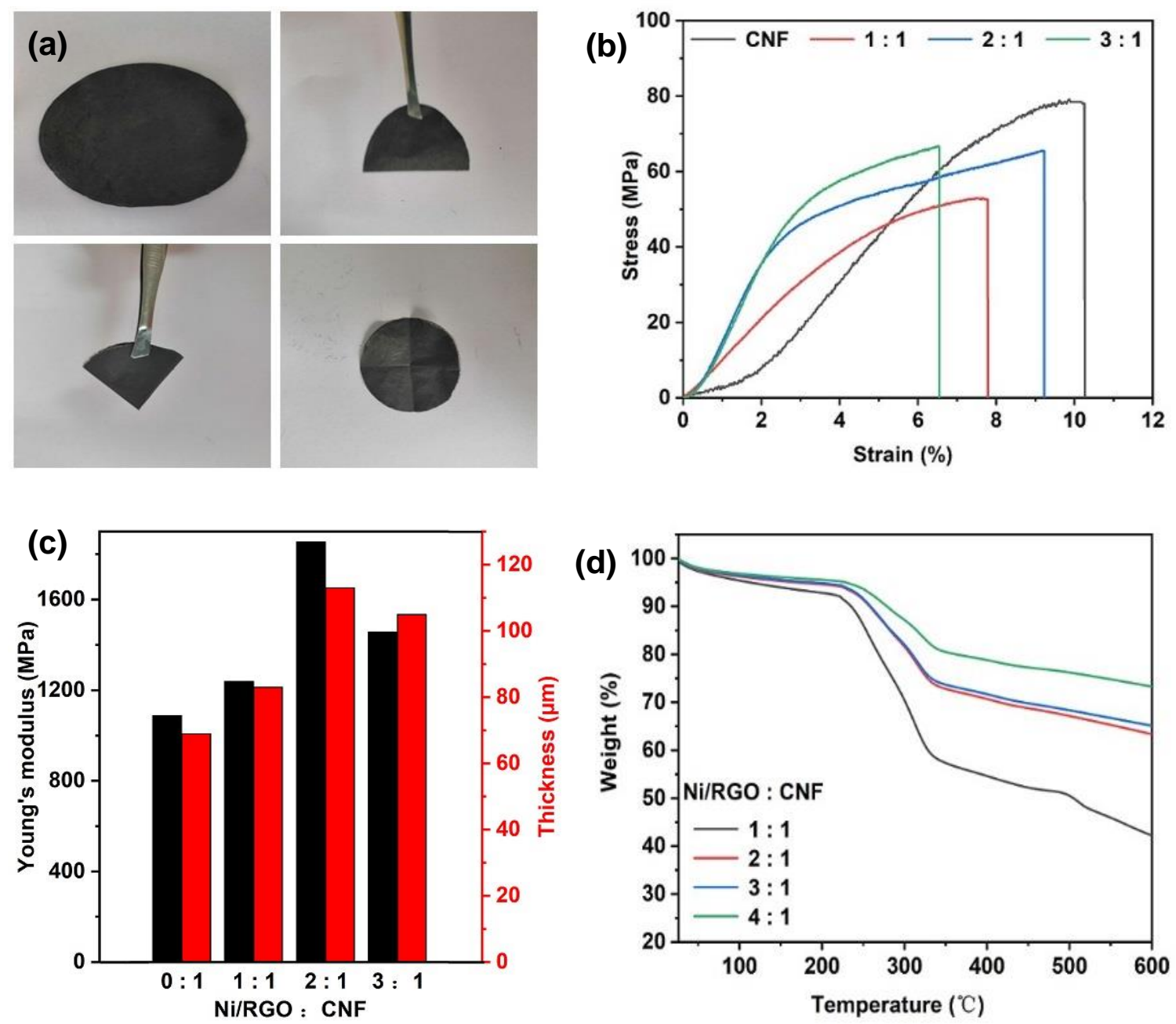

Fig. 3. (a) Photographs of Ni/RGO/CNF composite paper exhibiting high flexibility;

(b) Stress-strain curves of the Ni/RGO/CNF composite paper with various ratios of Ni/RGO and CNFs; (c) Thickness and Young's modulus with various ratios of Ni/RGO and CNFs; and (d) TG of the $\mathrm{Ni} / \mathrm{RGO} / \mathrm{CNFs}$ composite papers with various ratios of Ni/RGO and CNFs

To test the mechanical properties of the prepared composite paper, an electronic universal testing machine was used. As shown in Fig. 3a, due to the strengthening effect of adding $\mathrm{CNF}$, the $\mathrm{Ni} / \mathrm{RGO} / \mathrm{CNF}$ s composite paper (prepared with 2:1) displayed good strength and toughness, and no fractures or cracks were observed when the composite paper was folded. The mechanical property curves of pure CNFs and the composite paper with 
various ratios of Ni/RGO and CNFs are shown in Fig. 3b. The Ni/RGO/CNFs composite papers exhibited excellent integration of the mechanical strength and toughness. The pure CNF paper showed a tensile strength of $78.4 \mathrm{MPa}$, a fracture strain of $10.3 \%$, and a Young's modulus of $1088 \mathrm{MPa}$. With increasing content of $\mathrm{Ni} / \mathrm{RGO}$, the tensile strength and tensile fracture strain changed. The Young's modulus increased from $1088 \mathrm{MPa}$ to $1854 \mathrm{MPa}$, and then it decreased to $1457 \mathrm{MPa}$, as shown in Fig. 3c. The optimal mechanical properties of the composite paper were achieved when the ratio of Ni/RGO to CNFs was $2: 1$.

Thermal analysis of the composite paper was completed in air. As shown in Fig. 3b, with the increase of $\mathrm{Ni} / \mathrm{RGO}$ ratio in the composite paper, the residue char also gradually increased from $41.9 \%$ to $73.1 \%$ at $600{ }^{\circ} \mathrm{C}$, which showed that the thermal stability improved gradually. During the whole thermogravimetric analysis, the composite paper prepared with four different proportions degraded slowly at the beginning, then began to degrade rapidly at approximately $220{ }^{\circ} \mathrm{C}$, and the downward trend flattened at approximately $335{ }^{\circ} \mathrm{C}$. In addition, at the highest Ni/RGO ratio, the residual amount of the composite paper was also maximized, which indicated that the composite paper with a sandwich structure supported the superior flame retardancy.

\section{(a)}
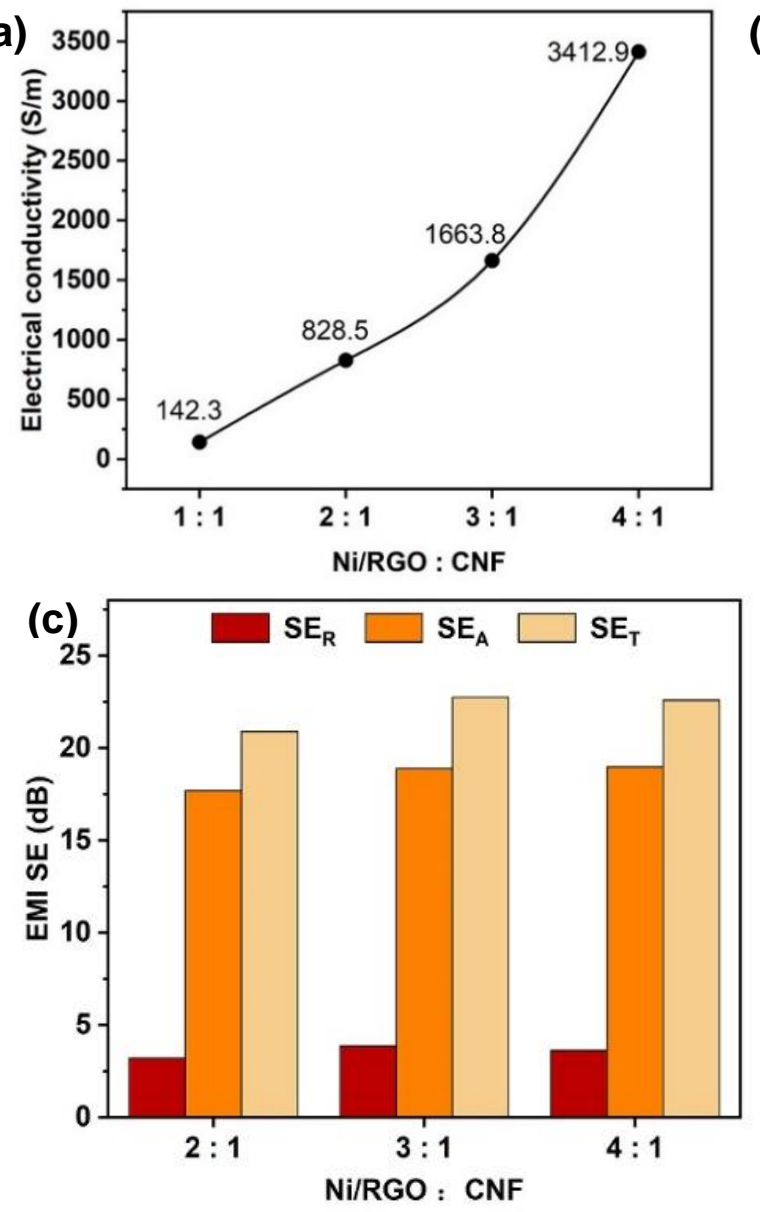

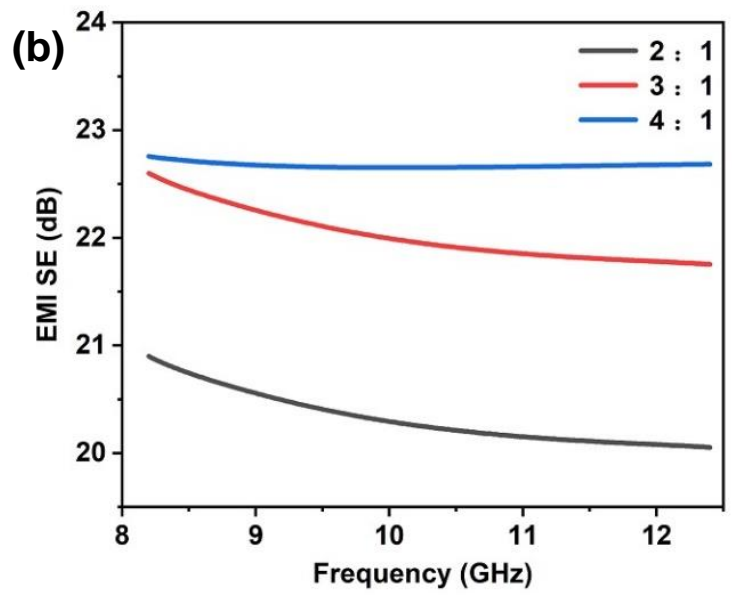

(d)

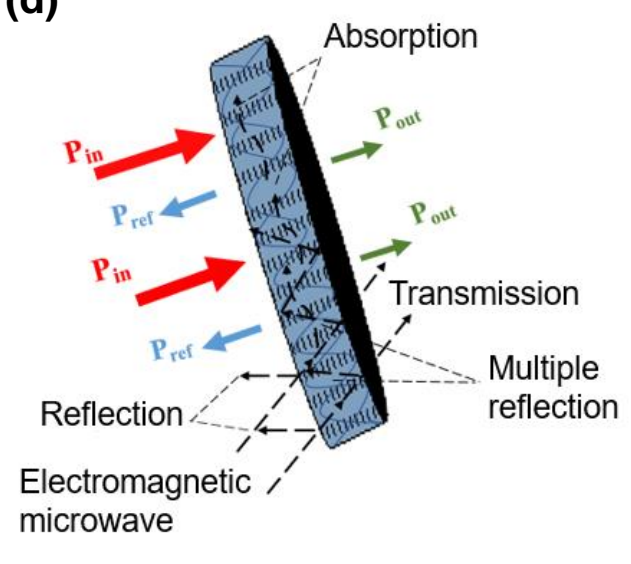

Fig. 4. (a) Plot of electrical conductivity; (b) Effect of Ni/RGO content on the EMI SE; (c) Comparison of SETotal, SEA, and SER at a frequency of $8.2 \mathrm{GHz}$; and (d) Schematic illustration of the electromagnetic wave transfer across the Ni/RGO/CNF composite paper with different ratios of $\mathrm{Ni} / \mathrm{RGO}$ and $\mathrm{CNFs}$ 
Electrical conductivity, the intrinsic ability to absorb electromagnetic energy, is important for EMI shielding materials, which can reflect the EMI shielding effectiveness to some extent. The as-prepared electrical conductivity of the composite paper for in-plane was higher than other composite papers obtained by a conventional mixing process. The composite paper with a ratio of $1: 1$ had an electrical conductivity of $142 \mathrm{~S} / \mathrm{m}$, which met the practical application requirement of $1.0 \mathrm{~S} / \mathrm{m}$ (Yang et al. 2017). When the ratio of $\mathrm{Ni} / \mathrm{RGO}$ in the $\mathrm{Ni} / \mathrm{RGO} / \mathrm{CNFs}$ composite paper was increased, the in-plane electrical conductivity of the composite paper also increased remarkably, as shown in Fig. 4a. The conductivity values were $828.5,1663.8$, and $3412.9 \mathrm{~S} / \mathrm{m}$, when the ratio of $\mathrm{Ni} / \mathrm{RGO}$ and CNFs was 2:1, 3:1, and 4:1, respectively. Moreover, the cross-plane electrical conductivity of $\mathrm{Ni} / \mathrm{RGO} / \mathrm{CNF}$ composite paper was low, but a conductive path was formed between, above, and below planes, which illustrated the effective penetration of $\mathrm{Ni} / \mathrm{RGO}$ into the CNF matrix during vacuum filtration. This phenomenon can be attributed to the onedimensional nanofibrous structure of CNFs with less insulating contact with 2D conductive nanosheets.

The EMI SE of composite papers in the microwave frequency range of $8.2 \mathrm{GHz}$ to 12.4 GHz (X-band) was measured. The as-prepared Ni/RGO/CNFs composite papers exhibited excellent EMI SE values. Figure $4 \mathrm{~b}$ reveals the variation of EMI SE of the composite papers with various ratios of $\mathrm{Ni} / \mathrm{RGO}$ and $\mathrm{CNF}$. Thus, it was concluded that the EMI SE curve did not substantially fluctuate within the measured frequency range, and with the increase of $\mathrm{Ni} / \mathrm{RGO}$ ratio, the EMI SE was improved. It was attributed to the formation of a good conductive network in the composite paper as the Ni/RGO ratio was increased.

When the ratio of $\mathrm{Ni} / \mathrm{RGO}$ to CNFs was $3: 1$, the $\mathrm{SE}$ of the composite paper reached $22.6 \mathrm{~dB}$ with a thickness of $105 \mu \mathrm{m}$, and the shielding efficiency reached $99.4 \%$, which means that most of the electromagnetic waves were shielded. Even if the ratio was at 2:1, the shielding performance reached up to $20.3 \mathrm{~dB}$. Thus, it met the requirements for commercial EMI shielding applications.

Figure $4 \mathrm{c}$ shows the total EMI shielding effectiveness $\left(S E_{\mathrm{T}}\right)$, microwave reflection $\left(S E_{\mathrm{R}}\right)$, and microwave absorption $\left(\mathrm{SE}_{\mathrm{A}}\right)$ at the frequency of $8.2 \mathrm{GHz}$. For example, when the ratio of Ni/RGO to CNF was 3:1, the $S E_{\mathrm{T}}, S E_{\mathrm{A}}$, and $S E_{\mathrm{R}}$ values were 22.6, 19.0, and 3.6 $\mathrm{dB}$, respectively. The $S E_{\mathrm{T}}$ is the sum of $S E_{\mathrm{A}}$ and $S E_{\mathrm{R}}$. Even if the content of $\mathrm{Ni} / \mathrm{RGO}$ or the thickness of composite paper changed, $S E_{\mathrm{A}}$ was much larger than $S E_{\mathrm{R}}$, which indicated an absorption-dominant shielding mechanism of the composite papers. There are three possible attenuation mechanisms during the electromagnetic shielding process, as shown in Fig. 4 d.

The $S E_{\mathrm{R}}$ is only attributed to the EM waves reflection of the surface of the composite papers, whereas $S E_{\mathrm{A}}$ is attributed to the CNF-supported Ni/RGO in the upper and lower layers of the composite paper to form a good conductive network, which consumes EM waves on its surface. In addition, because the composite paper has a layered structure, it plays an important role in multiple reflections, resulting in absorption and dissipation of EM waves. Therefore, $\mathrm{Ni} / \mathrm{RGO} / \mathrm{CNF}$ s composite paper with good electrical conductivity and shielding effect can meet the requirements of flexible EMI shielding materials and has a good application prospect. 


\section{CONCLUSIONS}

1. A convenient method was presented to fabricate $\mathrm{Ni} / \mathrm{RGO} / \mathrm{CNF}$ composite papers by a step-by-step pressure-assisted filtration. The lightweight and flexible nature of the composite papers thus developed indicated good electromagnetic wave absorption ability. The CNF played a good supporting role in the Ni/RGO film formation process.

2. Through changing the weight ratio of $\mathrm{Ni} / \mathrm{RGO}$ to CNFs in the composite paper, the ultimate tensile strength and fracture strain of the $\mathrm{Ni} / \mathrm{RGO} / \mathrm{CNFs}$ composite paper reached $78.4 \mathrm{MPa}$ and $10.3 \%$, respectively.

3. Because the prepared composite paper (3:1) with a thickness of $105 \mu \mathrm{m}$ exhibited excellent electrical conductivity up to $1664 \mathrm{~S} / \mathrm{m}$ and EMI SE of $22.6 \mathrm{~dB}$ at $8.2 \mathrm{GHz}$, it makes a good candidate for the EMI shielding and bio-based flexible electronic device.

\section{ACKNOWLEDGMENTS}

This work was supported by the special fund for Beijing Common Construction Project and Beijing Forestry University (Grant No. 2016HXKFCLXY001).

\section{REFERENCES CITED}

Bansala, T., Joshi, M., and Mukhopadhyay, S. (2019). "Electromagnetic interference shielding behavior of chemically and thermally reduced graphene based multifunctional polyurethane nanocomposites: A comparative study," Journal of Applied Polymer Science 136(25), Article ID 47666. DOI: 10.1002/app.47666

Bian, X. M., Liu, L., Li, H. B., Wang, C. Y., Xie, Q., Zhao, Q. L., Bi, S., and Hou, Z. L. (2017). "Construction of three-dimensional graphene interfaces into carbon fiber textiles for increasing deposition of nickel nanoparticles: Flexible hierarchical magnetic textile composites for strong electromagnetic shielding," Nanotechnology 28(4), Article ID 045710. DOI: 10.1088/1361-6528/28/4/045710

Cao, W. T., Chen, F. F., Zhu, Y. J., Zhang, Y. G., Jiang, Y. Y., Ma, M. G., and Chen, F. (2018). "Binary strengthening and toughening of MXene/cellulose nanofiber composite paper with nacre-inspired structure and superior electromagnetic interference shielding properties," ACS Nano 12(5), 4583-4593. DOI: 10.1021/acsnano.8b00997

Chen, Y., Zhang, H. B., Yang, Y. B., Wang, M., Cao, A. Y., and Yu, Z. Z. (2016). "Highperformance epoxy nanocomposites reinforced with three-dimensional carbon nanotube sponge for electromagnetic interference shielding," Advanced Functional Materials 26(3), 447-455. DOI:10.1002/adfm.201503782

Geim, A. K., and Novodelov, K. S. (2007). "The rise of graphene," Nature Materials 6(3), 183-191. DOI: 10.1038/nmat 1849

Hou, M. J., Xu, M. J., and Li, B. (2018). "Enhanced electrical conductivity of cellulose nanofiber/graphene composite paper with a sandwich structure," ACS Sustainable Chemistry and Engineering 6(3), 2983-2990. DOI: 10.1021/acssuschemeng.7b02683

Hsiao, S. T., Ma, C. C., Liao, W. H., Wang, Y. S., Li, S.-M., Huang, Y. C., Yang, R. B., and Liang, W. F. (2014). "Lightweight and flexible reduced graphene oxide/water- 
borne polyurethane composites with high electrical conductivity and excellent electromagnetic interference shielding performance," ACS Applied Materials and Interfaces 6(13), 10667-10678. DOI: 10.1021/am502412q

Huangfu, Y. M., Liang, C. B., Han, Y. X., Qiu, H., Song, P., Wang, L., Kong, J., Gu, J. W. (2019). "Fabrication and investigation on the Fe3O4/thermally annealed graphene aerogel/epoxy electromagnetic interference shielding nanocomposites," Composites Science and Technology 169, 70-75. DOI: 10.1016/j.compscitech.2018.11.012

Isogai, A., Saito, T., and Fukuzumi, H. (2011). "Tempo-oxidized cellulose nanofibers," Nanoscale 3(1), 71-85. DOI: 10.1039/c0nr00583e

Klemm, D., Kramer, F., Moritz, S., Lindstrom, T., Ankerfors, M., Gray, D., and Dorris A. (2011). "Nanocelluloses: A new family of nature-based materials," Angewandte Chemie-International Edition 50(24), 5438-5466. DOI: 10.1002/anie.201001273

Panigrahi, R., and Srivastava, S. K. (2015). "Trapping of microwave radiation in hollow polypyrrole microsphere through enhanced internal reflection: A novel approach," Scientific Reports 5, Article Number 7638. DOI: 10.1038/srep07638

Prasad, J., Singh, A. K., Shah, J., Kotnala, R. K., and Singh, K. (2018). "Synthesis of $\mathrm{MoS}_{2}$-reduced graphene oxide/ $\mathrm{Fe}_{3} \mathrm{O}_{4}$ nanocomposite for enhanced electromagnetic interference shielding effectiveness," Materials Research Express 5(5), 55028-55038. DOI: $10.1088 / 2053-1591 / \mathrm{aac} 0 \mathrm{c} 2$

Prasad, J., Singh, A. K., Haldar, K. K., Gupta, V., and Singh, K. (2019).

"Electromagnetic interference shielding effectiveness in 3D flower-like $\mathrm{MoS}_{2}$ rGO/gadolinium-doped nanocomposites," Journal of Alloys and Compounds 788, 861-872. DOI: 10.1016/j.jallcom.2019.02.246

Remyamol, T., John, H., and Gopinath, P. (2013). "Synthesis and nonlinear optical properties of reduced graphene oxide covalently functionalized with polyaniline," Carbon 59(4), 308-314. DOI: 10.1016/j.carbon.2013.03.023

Tian, K. H., Su, Z., Wang, H., Tian, X. Y., Huang, W. Q., and Xiao, C. (2017). 'N-doped reduced graphene oxide/waterborne polyurethane composites prepared by in situ chemical reduction of graphene oxide," Composites Part A: Applied Science and Manufacturing 94, 41-49. DOI: 10.1016/j.compositesa.2016.11.020

Xu, C., Wang, X., and Zhu, J. W. (2008). "Graphene-metal particle nanocomposites," Journal of Physical Chemistry C 112(50), 19841-19845. DOI: 10.1021/jp807989b

Xu, F., Chen, R. F., Lin, Z. S., Qin, Y. Y., Yuan, Y., Li, Y., Zhao, X., Yang, M. L., Sun, X. X., Wang, S. S., et al. (2018). "Superflexible interconnected graphene network nanocomposites for high-performance electromagnetic interference shielding," ACS Omega 3(3), 3599-3607. DOI: 10.1021/acsomega.8b00432

Yang, W. X., Zhao, Z. D., Wu, K., Huang, R., Liu, T. Y., Jiang, H., Chen, F., and Fu, Q. (2017). "Ultrathin flexible reduced graphene oxide /cellulose nanofiber composite films with strongly anisotropic thermal conductivity and efficient electromagnetic interference shielding," Journal of Materials Chemistry C 5(15), 3748-3756. DOI: $10.1039 / \mathrm{c} 7 \mathrm{tc00400a}$

Article submitted: August 8, 2019; Peer review completed: October 19, 2019; Revised version received and accepted: December 9, 2019; Published: December 11, 2019.

DOI: $10.15376 /$ biores.15.1.814-824 